\title{
Long-term cognitive, executive, and behavioral outcomes of moderate and late preterm at school age
}

\author{
Ju Hyun Jin, $\mathrm{MD}^{1, *}$, Shin Won Yoon, $\mathrm{MD}^{1}{ }^{1}$, Jungeun Song, MD, PhD², Seong Woo Kim, MD³ , Hee Jung Chung, MD, PhD ${ }^{1}$ \\ ${ }^{1}$ Department of Pediatrics, National Health Insurance Service Ilsan Hospital, Goyang, Korea; ${ }^{2}$ Department of Psychiatry, National Health Insurance Service Ilsan \\ Hospital, Goyang, Korea; ${ }^{3}$ Department of Physical Medicine and Rehabilitation, National Health Insurance Service Ilsan Hospital, Goyang, Korea
}

Background: There is increasing concern that moderate preterm (32-33 weeks' gestation) and late preterm (34-36 weeks' gestation) birth may be associated with minor neurodevelopmental problems affecting poor school performance.

Purpose: We explored the cognitive function, cognitive visual function, executive function, and behavioral problems at school age in moderate to late preterm infants.

Methods: Children aged $7-10$ years who were born at $32^{+0}$ to $36^{+6}$ weeks of gestation and admitted to the neonatal intensive care unit from August 2006 to July 2011 at the National Health Insurance Service Ilsan Hospital were included. We excluded children with severe neurologic impairments, congenital malformations, or chromosomal abnormalities. Neuropsychological assessments consisted of 5 neuropsychological tests and 3 questionnaires.

Results: A total of 37 children (mean age, $9.1 \pm 1.2$ years) participated. The mean gestational age at birth was $34.6 \pm 7.5$ weeks, while the mean birth weight was $2,229.2 \pm 472.8 \mathrm{~g}$. The mean full-scale intelligence quotient was $92.89 \pm 11.90 ; 24.3 \%$ scored between 70 and 85 (borderline intelligence functioning). An abnormal score was noted for at least one of the variables on the attention deficit hyperactivity disorder diagnostic system for $65 \%$ of the children. Scores below borderline function for executive quotient and memory quotient were $32.4 \%$ and $24.3 \%$, respectively. Borderline or clinically relevant internalizing problems were noted in $13.5 \%$ on the Child Behavior Check List. There were no significant associations between perinatal factors or socioeconomic status and cognitive, visual perception, executive function, or behavior outcomes.

Conclusion: Moderate to late preterm infants are at risk of developing borderline intelligence functioning and attention problems at early school age. Cognitive and executive functions that are important for academic performance must be carefully monitored and continuously followed up in moderate to late preterminfants.
Key words: Moderate preterm, Late preterm, Cognitive function, Executive function, Long-term outcome

\section{Key message}

Question: Infants born at moderate to late preterm gestations are known to have little problem later on, but is that really true? Finding: At school age, cognitive problem was observed in about a quarter of the children. In addition, more than half of the children was suspected of having attention problems.

Meaning: Moderate to late preterm infants are at risk of developing abnormal intelligence and attention problems at early school age, therefore they should not be neglected on longterm follow-up evaluation.

\section{Introduction}

Moderate preterm (32-33 weeks' gestation) and late preterm (34-36 weeks' gestation) births account for the major proportion of all preterm births, and these populations potentially have a large influence on public health. Because they are considered to be at low risk for abnormal neurodevelopmental outcomes and may not show signs of abnormal brain function in early infancy, they are usually not followed up properly. Major neurodevelopmental morbidities are usually detected during the first 2 years of life. Nevertheless, minor neurodevelopmental problems may not become apparent until school age, when more complex skills such as reading and writing are required for scholastic performance. ${ }^{1)}$ Previous studies demonstrated that premature children up to school age present with higher rates of difficulties with language skills, cognitive function, visuo-motor integration, and behavior adaptation than do their peers born at term. ${ }^{2,3)}$ There is increasing concern that moderate to late preterm birth is associated with long-term medical and behavioral morbidities with attention problems and impaired cognitive and academic performance at school age. ${ }^{4-11)}$ 


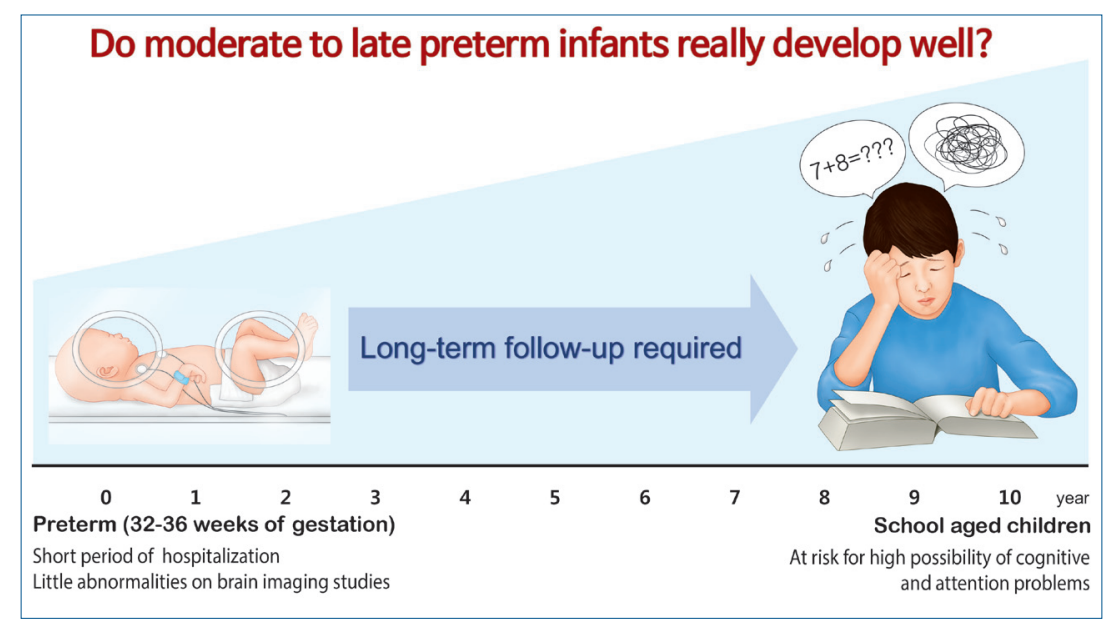

\section{Graphical abstract}

We aimed to evaluate neurodevelopmental outcomes including cognitive function, executive function, and emotional and behavioral development in school-aged children who were born moderate to late preterm, and who appear to be free of major neurological disabilities.

\section{Methods}

\section{Subjects}

Children aged $7-10$ years who were born at $32^{+0}-36^{+6}$ weeks of gestation and admitted to neonatal intensive care unit (NICU) during the period from August 2006 to July 2011 in the National Health Insurance Service Ilsan Hospital were eligible. During this period, a total of 1,398 neonates were born at our center, and 329 neonates were moderate to late preterm infants. Of these 329, 266 were admitted to the NICU (Fig. 1). Children with chromosomal abnormalities, congenital anomalies, and severe neurodevelopmental problems that would make it difficult to perform tests were excluded. After excluding 12 children, we randomly called 112 children who were living in 5 districts within $20-\mathrm{km}$ radius of the hospital, and 37 of them agreed to participate (Fig. 1). The main reasons for refusal were long distance due to moving, difficulty in making time to participate, and incorrect phone numbers. The children and at least one of the parents came together to the hospital and conducted the tests.

\section{General assessments}

Physical and neurological examinations were performed by 3 pediatricians. General information was obtained by questionnaires and maternal and clinical data from NICU were retrospectively reviewed from medical records.

\section{Neuropsychological assessments}

Five neuropsychological tests and 3 questionnaires were used in this study. All neuropsychological tests were performed by 2 well trained clinical psychologists.

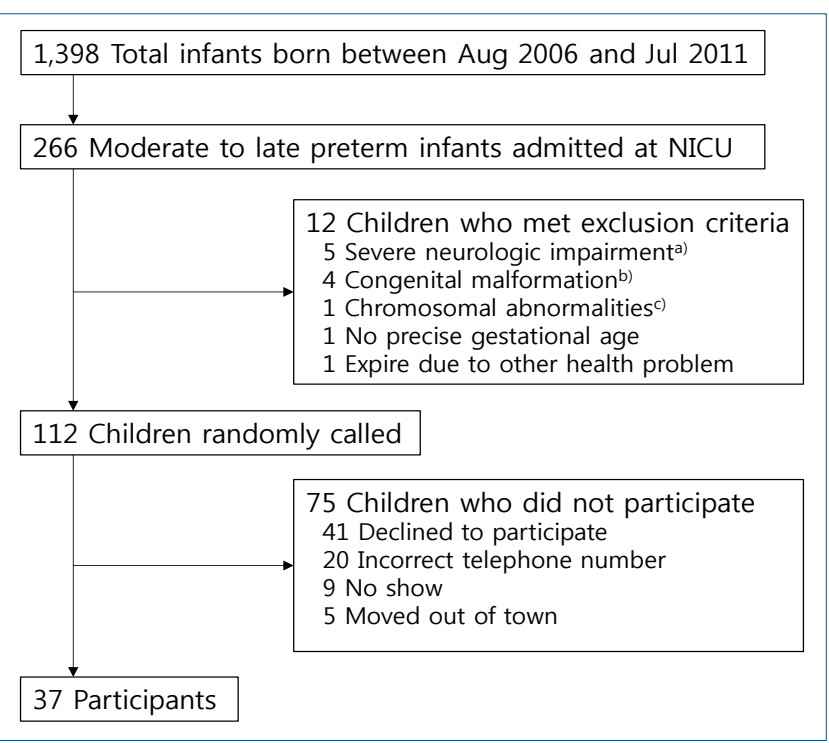

Fig. 1. Flow chart of the study participations. ${ }^{\text {a) }}$ Severe neurologic impairment includes epilepsy, cerebral palsy, and hypoxic ischemic encephalopathy. ${ }^{\text {b) }}$ Congenital malformation includes tracheoesophageal fistula, polydactyly, gastrointestinal tract malformation, and hypoplastic left heart syndrome. ${ }^{c)}$ Chromosomal abnormalities includes Down syndrome. NICU, neonatal intensive care unit.

\section{1) Cognitive function}

The Korean version of Wechsler Scale of Intelligence for Children - fourth edition (K-WISC-IV) was used. ${ }^{12)}$ Ten subsets provide a full-scale intelligence quotient (FSIQ) score and 4 index scores: verbal comprehension index (VCI), perceptual reasoning index (PRI), working memory index (WMI), and processing speed index (PSI). In addition to the 4 main index scores, 2 additional composite scores, the general abilities index (GAI) and the cognitive proficiency index (CPI), were used. The GAI is obtained by combining VCI and PRI index scores and is considered an alternate measure of global cognitive functioning that minimizes the impact of working memory and processing speed. The CPI composite index score is comprised of the WMI and PSI scores and measures efficient and proficient information processing. These scales have population means of 100 and standard deviations (SD) of 15 . Scores of 70-84 are considered borderline 
intelligence functioning, and scores $<70$ suggest intellectual disability.

\section{2) Cognitive visual function}

We used the Developmental Test of Visual Perception, 2nd edition (DTVP-2) ${ }^{13)}$ and the 3 rd edition (DTVP-3) ${ }^{14)}$ to assess general visual perception (GVP). This test is composed of 2 categories: motor-reduced visual perceptual (MRP) tasks and motorenhanced visual-motor tasks (visual-motor integration, VMI). A composite quotient score of $<80$ is considered abnormal.

\section{3) Attention, memory, and executive function}

The attention deficit hyperactivity disorder diagnostic system (ADS) is a computerized continuous performance test for diagnosis attention deficit hyperactivity disorder (ADHD). ${ }^{15)}$ Four test variables are recorded: omission errors, commission errors, reaction time, and reaction time variability. If there is at least one T-score $>70$ of the 4 variables, then ADHD is suspected. ${ }^{15}$ ) Memory was assessed using the Rey-Kim memory test. ${ }^{16)}$ Based on the scores of verbal and visual memory tests, a memory quotient (MQ) was calculated. Kim's Frontal-Executive Function Test was used to estimate executive quotient (EQ). ${ }^{17)}$ This test evaluates attention, language, visual-spatial function, and memory function.

\section{4) Emotional and behavioral problems}

The Child Behavior Check List (CBCL) is a widely used questionnaire to screen for emotional, behavioral, and social problems. ${ }^{18)}$ It measures T-scores for 8 behavioral problem scales that are combined to form broad-band scales of internalizing problems (anxious/depressed, somatic complaints, withdrawn subscales) and externalizing problems (aggression, rule-breaking behavior subscales). The Kovac's Children's Depression Inventory (CDI) is a psychological questionnaire that rates the severity of depression or dysthymic disorder. We used the Korean version CDI with cutoff points of scores of $>13 .{ }^{19)}$ The Korean version of a Revised Children's Manifest Anxiety Scale (RCMAS) to assess the level and nature of anxiety was used, in which total scores $>13$ are considered clinically significant. ${ }^{20)}$

\section{Statistical analyses}

Statistical analyses were performed using the SAS ver. 9.4 (SAS Institute, Cary, NC, USA). Categorical variables were expressed as number (\%) and continuous variables were expressed as mean \pm SD. We compared the scores of K-WISC-IV using the paired t test. The distributions of each index scores were represented by a box plot and the means were compared by repeated-measures analysis of variance. A $P$ value of $<0.05$ was considered statistically significant.

\section{Ethics statement}

This study was reviewed and approved by the Institutional Review Boards of the National Health Insurance Service Ilsan Hospital (approval number: 2017-63). Informed consent was obtained from the parents when they were enrolled.

\section{Results}

\section{Demographics and clinical characteristics}

Of the 37 children, 28 (75.7\%) were late preterm (34-36 weeks' gestation) and 9 (24.3\%) were moderate preterm (32-33 weeks' gestation) (Table 1). The mean gestational age at birth was $34.6 \pm 7.5$ weeks (range, $32^{+1}-36^{+3}$ weeks) and the mean birth weight was 2,229.2 $\pm 472.8 \mathrm{~g}$ (range, 1,100-3,110 g). The mean maternal age at delivery was $31.0 \pm 4.2$ years. Among the mothers, $70.3 \%$ were complicated pregnancy and $13.5 \%$ received antenatal steroids. Four children with respiratory distress syndrome were treated with surfactant and none had bronchopulmonary dysplasia. All children had one or more cranial ultrasound during admission and none had intraventricular hemorrhage of grade 3 or 4 .

There were no significant differences between infants who were enrolled $(n=37)$ and those who were not enrolled $(n=217)$ with respect to gestational age $(34.6 \pm 7.5$ vs. $34.6 \pm 9, P=0.812)$,

Table 1. Demographics and clinical characteristics $(n=37)$

\begin{tabular}{lc}
\hline Variable & Value \\
\hline Gestational age (wk) & $34.6 \pm 7.5\left(32^{+1}-36^{+3}\right)$ \\
Moderate preterm ( $\mathrm{n}=9)$ & $33.0 \pm 0.4$ \\
Late preterm ( $\mathrm{n}=28)$ & $35.0 \pm 0.7$ \\
Birth weight (g) & $2,229.2 \pm 472.8(1,100-3,110)$ \\
Male sex & $22(59.5)$ \\
Vaginal delivery & $12(32.4)$ \\
Apgar score $<7$ at 5 min & $5(13.2)$ \\
IUGR & $9(24.3)$ \\
Twins & $12 \pm 32.4$ \\
Maternal age at delivery (yr) & $31 \pm 4.2$ \\
Complicated pregnancy & $26(70.3)$ \\
IVF-ET & $8(21.6)$ \\
Antenatal steroid & $5(13.5)$ \\
Duration of hospitalization (day) & $16 \pm 10.1$ \\
Respiratory distress syndrome & $4(10.8)$ \\
Mechanical ventilator care & $6(16.2)$ \\
IVH grade 1-2 & $6(16.2)$ \\
IVH grade 3-4 & $0(0)$ \\
Child's age at test (yr) & $9.1 \pm 1.2(7.2-10.9)$ \\
Economic status & $12(32.4)$ \\
Poor & $19(52.8)$ \\
Average & $5(13.5)$ \\
Good & \\
Maternal education level & $17(45.9)$ \\
Completed high school or less & $20(54.1)$ \\
University or above & \\
Values are presented as mean $\pm S D($ range), mean SD, or number (\%). \\
SD, standard deviation; IUGR, intrauterine growth restriction; IVF-ET, in \\
aitro fertilization and embryo transfer; IVH, intraventricular hemorrhage. \\
mellitus, placenta abruptio, amniotic fluid problems, and fetal distress.
\end{tabular}




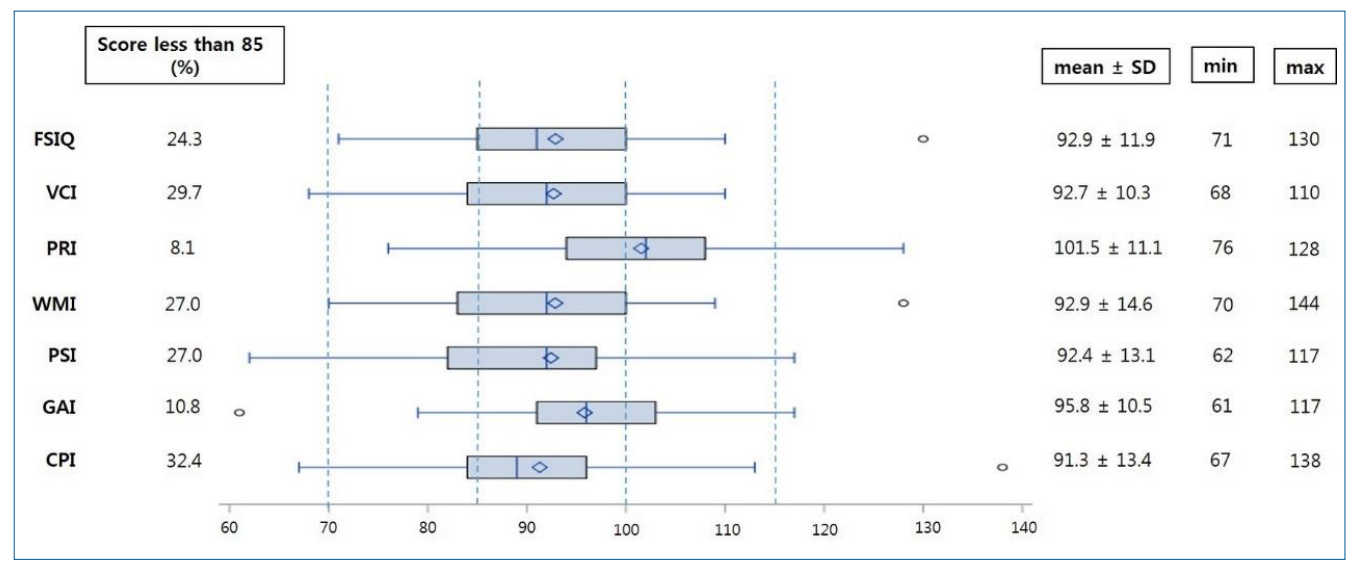

Fig. 2. Cognitive performance of the children (K-WISC-IV). K-WISC-IV, Korean Wechsler Intelligence Scale for Children - fourth edition; $\mathrm{FSIQ}$, full-scale intelligence quotient; VCI, verbal comprehension index; PRI, perceptual reasoning index; WMI, working memory index; PSI, processing speed index; GAI, general abilities index; $\mathrm{CPI}$, cognitive proficiency index; SD, standard deviation. Post hoc $P$ value of GPI vs. CPI was 0.037 . $P<0.05$ signifies statistical significance.

birth weight $(2,229.2 \pm 472.8$ vs. $2,273.5 \pm 412.0, P=0.557)$, respiratory distress syndrome $(10.8 \%$ vs. $14.3 \%, P=0.571)$, and duration of hospital days $(16 \pm 10.1$ vs. $14 \pm 7.2, P=0.324)$.

The mean age at examination was $9.1 \pm 1.2$ years (range, $7.2-$ 10.9 years). Eight children (21.6\%) wore glasses and corrected visual acuity were all $>0.8$. No child showed any deficit on neurological examination. One child had been treated for language delay. All children were attending regular classes at school and 6 children (16.1\%) answered the questionnaire that their academic performance was poor. Over half of mothers (54.1\%) had university education or above. Two-third of families (66.3\%) were of above average economic status.

\section{Cognitive function}

The results of K-WISC-IC showed a mean FSIQ of 92.89 \pm 11.90, a VCI of $92.70 \pm 10.29$, a PRI of $101.5 \pm 11.10$, a WMI of $92.86 \pm 14.61$, and a PSI of $92.43 \pm 13.12$ (Fig. 2). Although statistically insignificant, the mean FSIQ score was 4.9 points lower for moderate preterm children than for late preterm-born children. Although no child had intellectual disability (FSIQ $<70$ ), borderline intellectual functioning (FSIQ, 70-84) was observed in $24.3 \%$ of the children. Fig. 2 shows that children achieved lower score on VCI, WMI, and PSI than on the PRI $(P<$ 0.001). The mean GAI score $(95.84 \pm 10.52)$ was higher than the mean FSIQ with a difference of -2.95 , but this was not statistically significant $(P=0.053)$ (Table 2). The mean GAI score was higher than the mean CPI with a significant difference of $4.51(P=0.037)$ (Table 2).

\section{Cognitive visual function}

All children were normal in GVP on DTVP. The MRP score was abnormal in only 1 child (2.7\%): however, VMI score was abnormal in 5 children (13.5\%). Abnormal subset scores $(<5)$ on VMI were observed in $18.9 \%$ of the children.

\section{Attention, memory, and executive function}

The ADS test was performed in all children: however, 4 child.
Table 2. Mean differences in FSIQ-GAI, FSIQ-CPI, and GAI-CPI of the K-WISC-IV

\begin{tabular}{lcccc}
\hline Index & Mean & Mean difference value & $T$ value & $P$ value \\
\hline FSIQ - GAI & $92.89-95.84$ & -2.95 & -2 & 0.053 \\
FSIQ - CPI & $92.89-91.3$ & 1.6 & 1.42 & 0.163 \\
GAI - CPI & $95.84-91.3$ & 4.54 & 2.17 & 0.037 \\
\hline
\end{tabular}

Values of $P<0.05$ are statistically significant.

FSIQ, full-scale intelligence quotient; GAI, general ability index; CPI, cognitive proficiency index; K-WISC-IV, Korean Wechsler Intelligence Scale for Children - fourth edition.

ren who scored over 200 were deemed to have performed incorrectly and were excluded (Table 3 ). The proportion of children with abnormal T-scores $(\geq 70)$ in the ADS is shown in Table 3. In the visual ADS, $69.7 \%$ of the children showed abnormal results in at least one of the 4 variables. In the auditory ADS, $66.7 \%$ of children showed abnormal in at least 1 of 4 variables. Abnormal $(<70)$ or borderline scores (70-84) of MQ and EQ were 24.3\% and $32.4 \%$, respectively. Two children scored $<70$ on EQ and 3 children did so on MQ (Table 3).

\section{Emotional and behavioral problems}

On the CBCL, borderline clinically relevant internalizing problems were noted in $13.5 \%$ of the children, and $29.7 \%$ of the children had socialization problems. The mean CDI score was $3.86 \pm 4.23$ and $5.6 \%$ of the children scored more than 13 points. Using RCMAS, $18.9 \%$ of children were reported to be at risk for anxiety problems. The mean RCMAS score was $9.43 \pm 4.81$.

There were no significant associations with perinatal or socioeconomic factors and neuropsychological results. (post hoc $P$-values of FSIQ vs. economic status and FSIQ vs. maternal educational level were 0.7406 and 0.2483 , respectively.)

\section{Discussion}

Although the overall birth rate has been declining in Korea, the preterm birth rate has continued to increase and efforts have 
Table 3. Attention, memory, and executive function

\begin{tabular}{lc}
\hline Variable & No. $(\%)$ \\
\hline Attention deficit hyperactivity disorder diagnostic system $(\mathrm{n}=33)$ \\
Visual omission error $\geq 70$ & $14(42.4)$ \\
Visual commission error $\geq 70$ & $11(33.3)$ \\
Visual mean reaction time $\geq 70$ & $10(30.3)$ \\
Visual SD of reaction time $\geq 70$ & $14(42.4)$ \\
Auditory omission error $\geq 70$ & $13(39.4)$ \\
Auditory commission error $\geq 70$ & $4(12.1)$ \\
Auditory mean reaction time $\geq 70$ & $12(36.4)$ \\
Auditory SD of reaction time $\geq 70$ & $15(45.5)$ \\
Rey-Kim Memory Test & \\
MQ (n=37) & \\
$\leq 69$ (abnormal) & $3(8.1)$ \\
$70-84$ (borderline) & $6(16.2)$ \\
$85-115$ (average) & $24(64.9)$ \\
$\geq 115$ (superior) & $4(10.8)$ \\
Kim's Frontal Executive Function Neuropsychological Test & \\
EQ (n=37) & \\
$\leq 69$ (abnormal) & $2(5.4)$ \\
$70-84$ (borderline) & $10(27.0)$ \\
$85-115$ (average) & $23(62.1)$ \\
$\geq 115$ (superior) & $2(5.4)$ \\
\hline SD, standard deviation; MQ, memory quotient; EQ, executive quotient
\end{tabular}

been concentrated on improving survival rates and decreasing morbidity in high-risk extreme premature babies. However, since, moderate to late preterm babies account for the majority (85\%-90\%) of preterm births, ${ }^{21)}$ greater attention should be paid to the increasing risks of adverse long-term neurodevelopmental outcomes in late preterm-born babies. To our knowledge, this is the first report of neurodevelopmental outcomes of moderate to late preterm infants at school age in Korea.

We found that early school-aged children showed a lower mean FSIQ of $92.89 \pm 11.90$ compared to normal population mean of 100 . A sample size of 37 achieves $94 \%$ power to detect a difference of 7.1 between normal population mean of 100 and our participations mean of 92.9 with an estimated SD of 11.9 and with a significance level of 0.0500 using a 2-sided 1-sample $\mathrm{t}$ test. When analyzed categorically, borderline FSIQ (70-84) was found in $24.3 \%$ of the children. According to the distribution curve, borderline intellectual functioning is found in $13.6 \%$ of the normal population. ${ }^{22)} \mathrm{A}$ study of late preterm outcomes at 6 years of age showed that FSIQ of late preterm was 100.58 , lower than FSIQ of full-term control 101.94, with FSIQ scores $<85$ in $21 \%$ of late preterm versus $12 \%$ in full-term children (odds ratio, 2.17). ${ }^{4)}$

Our results are comparable with those of a Korean study (mean gestational age, 30.6 \pm 3.2 weeks) with no major neurodevelopmental impairments, in which $27.5 \%$ of children were with FSIQ $<85 .{ }^{23)}$ Because data for late preterm children are scarce, our data showing $24.3 \%$ of children with FSIQ $<85$ add to the current knowledge, to the effect that moderate to late preterm infants are considered "at-risk" rather than "low-risk" in terms of cognitive outcomes. ${ }^{5,24,25)}$ Because intelligence quotient (IQ) is thought to be an important predictor of social and vocational outcome, late preterm infants with lower IQ may grow up with lower levels of educational achievement and income. ${ }^{26)}$ In other studies of moderate to late preterm infants, children were at greater risk for poor school outcome than were full-term infants, with lower reading and math scores. ${ }^{27,28)}$ The brain almost doubles in size between 32-36 weeks' gestation as differentiation proceeds throughout the cortex and myelination continues. $\left.{ }^{6}\right)$ Preterm birth with exposure to the extrauterine environment may increase the risk for disruption of growth and development in the vulnerable preterm brain.

Cognitive impairment is a strong predictor of ADHD symptoms in extremely preterm-born children with minor neurodevelopmental impairments. ${ }^{29)}$ Our data showed that mean GAI was significantly higher than mean CPI with a difference of 4.51. A GAI-CPI discrepancy (GAI>CPI), meaning weakness in information processing, is frequently found in $\mathrm{ADHD}$ patients. 23,30,31) Our ADS data also showed that more than half of the children had at least one abnormal T-score on the visual or auditory testing. Since abnormal scores are expected in $2.3 \%$ of normal children, much higher percentage of abnormal scores in our study suggest increased risk for attention--deficit problem. ${ }^{15)}$ Our findings are consistent with those of a previous report that showed even preterm infants with a low risk of neurodevelopmental deficits achieved lower scores on attention tasks. ${ }^{32}$ The attention problems in moderate preterm children indicate regulation difficulties that may underlie their cognitive and behavioral problems. ${ }^{6}$ Premature birth causing early brain development disturbances and their related treatment may have contributed to this outcome, in addition to the underlying causes of premature birth or epigenetic processes. ${ }^{5)}$ Prematurity induces alterations of white matter connectivity for visual information processing in dorsolateral systems, which are neural networks responsible for coordination of goal-directed behavior. ${ }^{33,34)}$

Below borderline scores of MQ in 24.3\% and EQ in 32.4\%, further suggest the existence of impaired executive function in moderate to late preterm children. ${ }^{35}$ Below borderline score was reported in $15.9 \%$ of normal children population. ${ }^{16,17)}$ Strong correlations have been shown between executive functions and academic achievement and behavioral functioning. ${ }^{3)}$ Because deficits in executive function in early childhood persist into adulthood, longitudinal follow-up may facilitate early detection and timely intervention.

We found increased risk for anxiety problems (18.9\%) on RCMAS and slightly increased internalization problems (13.5\%) on the CBCL. Although we did not have full-term control group, it is worthwhile comparing several studies suggesting increased risk of developmental and behavioral problems in late preterm infants, especially as they enter school. ${ }^{4-7)}$ In a study using the CBCL, 23.3\% of preterm infants (26-33 weeks' gestation) in a brain-sparing group had behavioral and attention problems at the age of 11 years. ${ }^{36}$ A follow-up study with questionnaire data of 7-year-old children born at 32-35 weeks of gestation 
showed that almost one-third of the children had learning difficulties, and $19 \%$ of them showed an abnormal hyperactivity score when the population norm was $10 \% .^{5)} \mathrm{A}$ Korean study of preterm birth with no major neurodevelopmental impairments also reported $14.0 \%-26.7 \%$ of behavioral problems on the CBCL. ${ }^{23)}$ Interruption of maturation processes of the brain, higher morbidity in premature infants, and treatment procedures in the NICU have negative impacts and can amplify long-term consequences on behavior and quality of life of children born late preterm.

Our study has some limitations that suggest the need for more research in this area. Our study included a relatively small number of children from a single center. There is also the possibility that parents who agreed to participate may have had concerns regarding their children's development and behavior, and that participants may therefore have been skewed toward children who were not doing well at school. Another limitation is that we did not have a full-term born control cohort for comparison.

A major strength of our study is that we investigated a wide range of neurodevelopmental outcomes, including various domains of cognitive and executive function in school-aged children using direct, objective, standardized assessments.

In conclusion, moderate to late preterm infants are at risk for developing borderline intelligence at school age. Executive function deficits, especially attention problems, were prominent. Our study supports the importance of periodic assessments and long-term follow-up in moderate to late preterm children, even if they have no signs of disabilities in early life, as these subtle or minor problems tend to become more obvious as the child grows to school age. Because minor neurodevelopmental problems such as borderline intelligence, and behavioral and adaptive functioning problems are more responsive to early interventions, ${ }^{23)} \mathrm{closer}$ follow-up monitoring of neurodevelopment after discharge for early detection and referral to early intervention is needed. The impact of this large and growing population of moderate to late preterm infants should not be underestimated or neglected on long-term follow-up evaluation.

\section{Conflicts of interest}

No potential conflict of interest relevant to this article was reported.

\section{Acknowledgments}

This study was supported by the National Health Insurance Service Ilsan Hospital research fund of 2017 (IRB approval No. 2017-63). We acknowledge the valuable support of Jung Hwa Hong (Research and analysis team, National Health Insurance Service Ilsan Hospital) in the statistical analysis for this study.

See the commentary "What do we know about the long-term cognitive and behavioral outcomes of school-aged children who were born moderate to late preterm?" via https://doi.org/ 10.3345/cep.2019.01340.
See the commentary "Should we regularly evaluate the neurodevelopmental status of moderate and late preterm infants?" via https://doi.org/10.3345/cep.2020.00472.

\section{References}

1. Voss W, Neubauer AP, Wachtendorf M, Verhey JF, Kattner E. Neurodevelopmental outcome in extremely low birth weight infants: what is the minimum age for reliable developmental prognosis? Acta Paediatr 2007; 96:342-7.

2. Foulder-Hughes LA, Cooke RW. Motor, cognitive, and behavioural disorders in children born very preterm. Dev Med Child Neurol 2003; 45:97-103.

3. Lee HJ, Park HK. Neurodevelopmental outcome of preterm infants at childhood: cognition and language. Hanyang Med Rev 2016;36:55-8.

4. Talge NM, Holzman C, Wang J, Lucia V, Gardiner J, Breslau N. Late-preterm birth and its association with cognitive and socioemotional outcomes at 6 years of age. Pediatrics 2010;126:1124-31.

5. McGowan JE, Alderdice FA, Holmes VA, Johnston L. Early childhood development of late-preterm infants: a systematic review. Pediatrics 2011; 127:1111-24.

6. van Baar AL, Vermaas J, Knots E, de Kleine MJ, Soons P. Functioning at school age of moderately preterm children born at 32 to 36 weeks' gestational age. Pediatrics 2009;124:251-7.

7. Huddy CL, Johnson A, Hope PL. Educational and behavioural problems in babies of 32-35 weeks gestation. Arch Dis Child Fetal Neonatal Ed 2001;85:F23-8.

8. Chan E, Quigley MA. School performance at age 7 years in late preterm and early term birth: a cohort study. Arch Dis Child Fetal Neonatal Ed 2014;99:F451-7.

9. Cserjesi R, Van Braeckel KN, Butcher PR, Kerstjens JM, Reijneveld SA, Bouma A, et al. Functioning of 7-year-old children born at 32 to 35 weeks' gestational age. Pediatrics 2012;130:e838-46.

10. Odd DE, Emond A, Whitelaw A. Long-term cognitive outcomes of infants born moderately and late preterm. Dev Med Child Neurol 2012; 54:704-9.

11. de Jong M, Verhoeven M, van Baar AL. School outcome, cognitive functioning, and behaviour problems in moderate and late preterm children and adults: a review. Semin Fetal Neonatal Med 2012;17:163-9.

12. Kwak GJ, Oh SH, Kim CT. K-WISC-IV manual for professionals. 4th ed. Seoul: Hakjisa Publisher, 2011.

13. Hammill DD, Pearson NA, Voress JK, Frostig M. Developmental test of visual perception: DTVP-2. 2nd ed. Austin (TX): Pro-Ed, 1993.

14. Hammill DD, Pearson NA, Voress JK, Frostig M. Developmental test of visual perception: DTVP-3. 3rd ed. Austin (TX): Pro-Ed, 2014.

15. Shin MS, Cho SZ, Chun SY, Hong KE. A study of the development and standardization of ADHD diagnostic system. Korean J Child Adol Psychiatr 2000;11:91-9.

16. Kim HG. Children's Rey-Kim memory test: manual. Daegu: Neuropsy Pub, 2005.

17. Kim HG. Children's kims frontal-executive function test; manual. Daegu: Neuropsy Pub, 2005.

18. Achenbach TM, Edelbrock CS. Manual for the child behavior checklist and revised child behavior profile. Burlington (VT): University of Vermont, Department of Psychiatry, 1983.

19. Cho SC, Lee YS. Development of the Korean form of the Kovacs' children's depression inventory. J Korean Neuropsychiatr Assoc 1990; 29:943-56

20. Choi JS, Cho SC. Assessment of anxiety in children: reliability and validity of revised children's manifest anxiety scale. J Korean Neuropsychiatr Assoc 1990;29:691-702.

21. Korean Statistical Information Service [Internet]. Daejeon (Korea): Statistics Korea; 2018 [cited 2019 Sep 20]. Available from: http://kosis. $\mathrm{kr} / \mathrm{eng} /$ statisticsList/statisticsListIndex.do?menuId=M_01_01\&vwcd= 
MT_TITLE\&parmTabId=M_01_01.

22. Wieland J, Zitman FG. It is time to bring borderline intellectual functioning back into the main fold of classification systems. BJPsych Bull 2016; 40:204-6.

23. Joo JW, Choi JY, Rha DW, Kwak EH, Park ES. Neuropsychological outcomes of preterm birth in children with no major neurodevelopmental impairments in early life. Ann Rehabil Med 2015;39:676-85.

24. Fan RG, Portuguez MW, Nunes ML. Cognition, behavior and social competence of preterm low birth weight children at school age. Clinics (Sao Paulo) 2013;68:915-21.

25. Palumbi R, Peschechera A, Margari M, Craig F, Cristella A, Petruzzelli MG, et al. Neurodevelopmental and emotional-behavioral outcomes in late-preterm infants: an observational descriptive case study. BMC Pediatr 2018;18:318.

26. Moster D, Lie RT, Markestad T. Long-term medical and social consequences of preterm birth. NEngl J Med 2008;359:262-73.

27. Chyi LJ, Lee HC, Hintz SR, Gould JB, Sutcliffe TL. School outcomes of late preterm infants: special needs and challenges for infants born at 32 to 36 weeks gestation. J Pediatr 2008;153:25-31.

28. Lipkind HS, Slopen ME, Pfeiffer MR, McVeigh KH. School-age outcomes of late preterm infants in New York City. Am J Obstet Gynecol 2012; 206: 222.e1-6.

29. Elgen SK, Sommerfelt K, Leversen KT, Markestad T. Minor neurodevelopmental impairments are associated with increased occurrence of
ADHD symptoms in children born extremely preterm. Eur Child Adolesc Psychiatry 2015;24:463-70.

30. Goo MJ, Oh SW, Lee SY, Paik YS, Lee JH, Hwang KS. Cognitive characteristics of attention-deficit hyperactivity disorder in Korean Wechsler Intelligence Scale for Children-Fourth Edition: focused on general ability index and cognitive proficiency index. J Korean Acad Child Adolesc Psychiatry 2016;27:313-8.

31. Cornoldi C, Giofrè D, Orsini A, Pezzuti L. Differences in the intellectual profile of children with intellectual vs. learning disability. Res Dev Disabil 2014;35:2224-30.

32. Caravale B, Tozzi C, Albino G, Vicari S. Cognitive development in low risk preterm infants at 3-4 years of life. Arch Dis Child Fetal Neonatal Ed 2005;90:F474-9.

33. Fazzi E, Bova SM, Uggetti C, Signorini SG, Bianchi PE, Maraucci I, et al. Visual-perceptual impairment in children with periventricular leukomalacia. Brain Dev 2004;26:506-12.

34. Atkinson J, Braddick O. Visual and visuocognitive development in children born very prematurely. Prog Brain Res 2007;164:123-49.

35. Taylor HG, Clark CA. Executive function in children born preterm: risk factors and implications for outcome. Semin Perinatol 2016;40:520-9.

36. van den Broek AJ, Kok JH, Houtzager BA, Scherjon SA. Behavioural problems at the age of eleven years in preterm-born children with or without fetal brain sparing: a prospective cohort study. Early Hum Dev 2010;86:379-84. 